\title{
Visualização Postural Bidimensional utilizando Sensor de Profundidade de Baixo Custo
}

\author{
Danilo Borges da Silva ${ }^{1}$ \\ ${ }^{1}$ Universidade Estadual do Paiuí (UESPI) \\ daniloefrn.uespi.br
}

\begin{abstract}
Postural assessment is the main method for evaluating, diagnosing, and treating postural deviations caused by several reasons, whether natural or irregular posture. In this paper, we used the Kinect sensor and Unity to collect data and perform a postural reading of standing up individuals in their natural position and observe possible deviations through angular measurements of predefined joints. In the results, the proposed method was compared with the manual evaluation technique that uses a symmetrograph. We show that the method can be used to evaluate postures as an alternative to the symmetrograph.
\end{abstract}

Resumo. A avaliação postural consiste de um método para avaliar, diagnosticar e tratar os desvios de postura influenciados por diversos fatores, sejam eles congênitos ou pelo mal posicionamento. Neste trabalho, utilizou-se o sensor Kinect juntamente com a Unity para coletar dados e realizar a leitura postural de indivíduos em pé, na sua posição natural, e observar os possíveis desvios encontrados por meio das medições angulares de articulações pré-definidas. Nos resultados, o método proposto foi comparado com a técnica de avaliação que utiliza um simetrógrafo. Concluiu-se mostrando que o método pode vir a ser utilizado para avaliar posturas como alternativa ao simetrógrafo.

\section{Introdução}

Com o crescimento do uso de aparelhos eletrônicos portáteis, principalmente de celulares e computadores/laptops, os riscos de problemas de saúde relacionados à postura têm se agravado. Esses problemas aparecem ao longo da vida provocando outra série de doenças, entre elas a dor de coluna. Desde 2010, estudos realizados apontam a dor lombar como uma doença prioritária da OMS (Organização Mundial de Saúde) e destacada como a maior contribuinte para a incapacidade em todo o mundo [Hoy 2014]. Além disso a OMS estima que mais de $80 \%$ das pessoas terão pelo menos duas crises relacionadas com a dor de coluna durante a vida.

Para evitar a contração de problemas relacionados com a postura o melhor tratamento é a prevenção. Entretanto, como esses problemas não recebem a devida atenção por programas de prevenção, nas secretarias de saúde no Brasil, acabam passando despercebidas pela comunidade até serem deflagradas. Sendo assim, é preciso ficar atento a postura em ações como sentar, andar e se movimentar; caso dores sejam percebidas devese contactar imediatamente um profissional. O profissional mais indicado é fisioterapeuta que irá diagnosticar a postura por meio da realização de exames posturais e indicar o melhor tratamento. 
Este trabalho está inserido no contexto de examinar a postura. O principal objetivo é verificar se é possível fazer a mensuração postural por meio de um sensor de profundidade de baixo custo. Atualmente, uma forma de mensuração bastante utilizada é a fotogrametria; em contra partida, o método aqui proposto utiliza um sensor de profundidade (câmera 3D) que promove a captura da postura em qualquer posição possibilitando maior capacidade de análise da postura.

Para isso, utilizou-se a plataforma Unity, mundialmente conhecida para criação de jogos, em conjunto com o sensor Kinect, uma câmera que capta profundidade. A base lógica do método proposto é a computação gráfica com geometria analítica para transformar a postura tridimensional (3D) captada pelo Kinect em visões bidimensionais da postura do usuário em dois planos: frontal e sagital. Essas visões são as utilizadas por técnicas clássicas de avaliação postural.

O método proposto foi comparado com técnica clássica que mensura a postura utilizando o simetrógrafo com manipulação manual ou recorrendo ao uso de imagens (fotogrametria). Nessa comparação, o método mostrou-se uma excelente alternativa para mensurar a postura, possibilitando maior liberdade do usuário (paciente), pois tanto a captura da postura quanto o cálculo das angulações de algumas juntas foram realizadas automaticamente.

\section{Trabalhos Relacionados}

Nesta seção, são apresentados trabalhos que fizeram uso do sensor de profundidade de baixo custo conhecido como Kinect para vários fins e trabalhos que mostram técnicas de mensuração postural comparáveis a que será apresentada na Seção 3.

O sensor de profundidade Kinect 360 da Microsoft tem sido utilizado em pesquisas envolvendo visão computacional, realidade virtual e aumentada, animação e interatividade. Por ser um equipamento de baixo custo apresenta um ruído (na captura) considerável que varia de acordo com a aplicação [Andersen et al. 2012]. Neste sentido, os pesquisadores utilizam técnicas para tratamento dos dados capturados pelo sensor com o intuito de diminuir o ruído e problemas na oclusão das juntas [Shum et al. 2013]. Neste trabalho, utilizou-se o tratamento das posturas capturadas no plano de projeção, em cada frame, com o intuito de tratar desregularidades apresentadas pelo sensor na visualização.

Embora existam ruídos consideráveis, os sistemas de captura de movimento de baixo custo estão sendo utilizados no campo da área da saúde com aplicações na reabilitação. [de Gusmão Lafayette et al. 2019] realizaram um estudo para calcular a discrepância de membros inferiores utilizando análise dos dados obtidos com o sensor de captura. E [Xu et al. 2020] utilizaram um sensor Kinect para observar deformações na coluna. O método que será apresentado pode vir a ser utilizado por um profissional de fisioterapia como um equipamento para auxílio clínico e de baixo custo, podendo ser usado para o acompanhamento da evolução do paciente ao longo de um tratamento.

A avaliação postural utilizada por fisioterapeutas baseia-se, em sua maioria, na análise visual por meio da observação das curvaturas da coluna vertebral e por assimetrias corporais no plano sagital e frontal [dos Santos 2018]. Para isso são utilizados equipamentos como o simetrógrafo e observado o paciente nesses dois planos (Figura 1). Outro método utiliza fotogrametria computadorizada, com auxílio de etiquetas autoadesivas para marcar pontos de interesse [Iunes et al. 2009]. Neste trabalho, os pontos (juntas) 

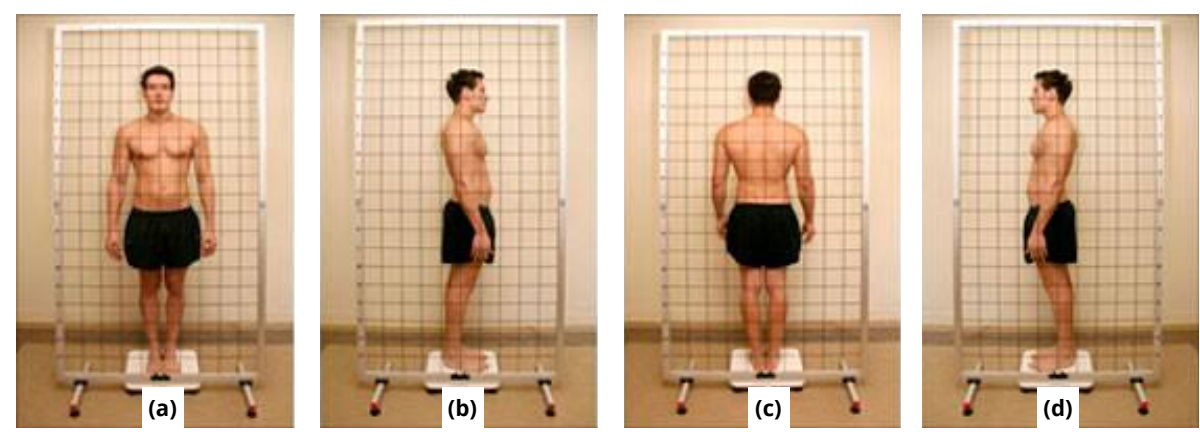

Figura 1. Avaliação postural com auxílio do simetrógrafo. São observados a postura no plano de projeção frontal, (a) e (c), e plano sagital, (b) e (d). fonte: https://bit.ly/3eHi6Sz

de interesse são automaticamente capturados por um algoritmo e os planos de projeção são criados em tempo real, de acordo com as posições das juntas, dispensando o uso do simetrógrafo. Na seção a seguir será descrito o método proposto.

\section{Método Proposto}

Neste trabalho, utilizou-se um Sensor Microsoft Kinect (versão XBOX 360) para capturar a postura do usuário - conjunto das posições de todas as principais juntas adquiridas pelo sensor em um instante de tempo. Para isso, foi utilizada a biblioteca Kinect SDK, versão 1.8, que se comunica com o sensor. Por meio dessa biblioteca é possível capturar a postura do usuário utilizando algoritmos implementados em C\#, por exemplo.

Como base da implementação do ambiente foi utilizado um asset chamado Kinect with MS-SDK presente na Asset Store da Unity que implementa a conexão com o Kinect SDK [Rumen 2013]. Com base nesse asset, e nos cenários (scenes) produzidos pelo mesmo, realizou-se uma pesquisa investigativa em seu código e extraíram-se as informações necessárias para formular o método proposto que consiste em capturar a postura e realizar a visualização dos dados capturados em duas visões bidimensionais para fins de verificação postural humana. Para isso, foram realizadas as seguintes atividades:

- Captura da Postura (Subseção 3.1): foram extraídas as posições tridimensionais (3D) de cada junta do usuário e armazenadas em um arquivo (csv) utilizando a base da implementação.

- Visualização 3D da Postura (Subseção 3.2): foi criada uma scene (cena) para visualizar os dados capturados no arquivo e rotacionar a câmera livremente, em cada instante de tempo.

- Visualização 2D da Postura (Subseção 3.3): foi criada uma scene para visualizar os dados capturados em 3D em projeções bidimensionais; da mesma forma como é feita em uma avaliação postural com o simetrógrafo que utiliza dois planos de visualização: o frontal e o sagital.

- Cálculo dos Ângulos de Interesse (Subseção 3.4): foram calculados os ângulos a partir das juntas de interesse nos planos de visão distintos para visualização.

Nas subseções a seguir são descritos os procedimentos realizados que em conjunto compõem o método proposto. A visão geral do método pode ser observada na Figura 2 (graphical abstract). Foram utilizadas três cenas (scenes), uma dessas é uma cena 


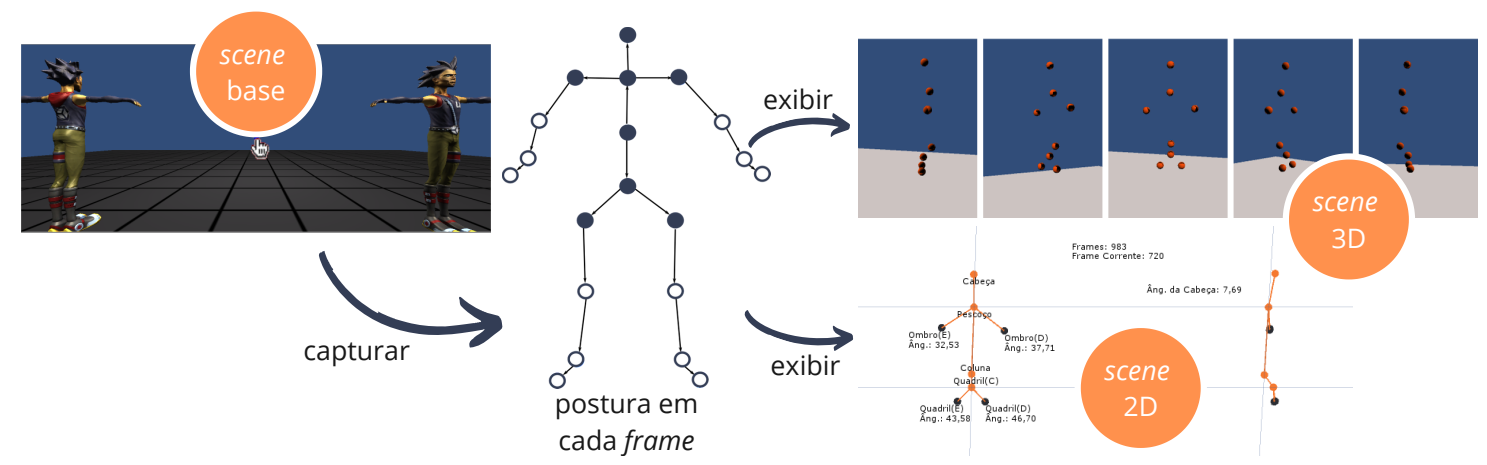

Figura 2. Esquema do método proposto. Os círculos, em laranja, representam as cenas utilizadas no método. As setas são as ações realizadas que manipulam as posturas capturadas em cada frame do usuário. Na postura foram exibidas e tratadas somente as juntas destacadas em azul para as visualizações 2D e 3D, as demais juntas foram desconsideradas.

produzida pela base de implementação com um algoritmo de armazenamento (1), e as outras cenas foram criadas para fazer a leitura do arquivo, criado em (1), e processados de acordo com o objetivo de visualização.

\subsection{Captura da Postura}

Para realizar a captura da postura do usuário e posterior armazenamento criou-se um arquivo no formato CSV. Nesse arquivo foram capturadas todas as posições das juntas adquiridas pelo Sensor Kinect, exibidos no esquema do personagem em azul na Figura 2. As posições são vetores tridimensionais que em conjunto determinam a posição das juntas (20) capturadas em um frame. Sendo assim, para cada instante de tempo existem posições armazenadas correspondendo a cada junta.

Entre essas posições, este trabalho limitou-se a um escopo formado por oito pontos de interesse: cabeça, pescoço, ombros (direito e esquerdo), coluna, quadril (centro), quadril direito e quadril esquerdo. Objetivando a realização de uma análise de correspondência à visualização de projeção planar frontal e sagital. Essas juntas tratam-se dos principais pontos de interesse que os profissionais da área, fisioterapeutas, utilizam para correlacionar os pontos anatômicos da postura de uma pessoa [de Matos 2011].

\subsection{Visualização 3D da Postura}

Para visualizar a postura tridimensional do usuário criou-se uma cena tridimensional com os oito pontos de interesse. Em cada junta de interesse foi desenhado uma esfera (Figura 2, scene 3D). Nessa cena, ao iniciar, o algoritmo lê o arquivo de armazenamento e transfere a posição de cada junta em um vetor (array) de tamanho três para capturar a posição, isso para cada ponto. No vídeo de referência (https : / / youtu . be / j7b IuHXaBQY) pode-se observar este scene.

O principal objetivo dessa scene é perceber que as posições capturadas não estão totalmente alinhadas, principalmente o quadril, mostrando que deve-se realizar um tratamento para que possa ser comparado a visão de projeção independentemente do frame. A ideia é produzir essa visão de projeção de forma estática, ou seja, mesmo com o usuário se movimentando será possível destacar os dois planos de projeção tratados com o uso do simetrógrafo, em cada instante de tempo. 


\subsection{Visualização 2D da Postura}

Na Seção 2, observou-se que uma grade (simetrógrafo) é usada com o objetivo de "projetar" o paciente na mesma e assim poder aferir a altura das juntas e as variações angulares em duas visões: sagital e frontal. Neste contexto, foi preciso utilizar a pose do usuário tridimensional capturada e projetar em dois planos. Isso pode ser realizado utilizando geometria analítica com o cálculo da projeção ortogonal, desde que os planos estejam bem definidos. A seguir é mostrado como foi realizada a criação do plano de projeção frontal da postura do usuário, que originalmente não está alinhado na captura, e a projeção dos pontos presentes neste plano no plano de visualização que é observado na scene 2D (Figura 2).

Para criar o plano frontal $\left(\pi_{f}\right)$ foram utilizados três pontos: a posição central do quadril ( $\left.p_{\text {hip_center }}\right)$, a posição do quadril direito $\left(p_{\text {hip_right }}\right)$ e a posição do quadril esquerdo $\left(p_{\text {hip_left }}\right)$. Visto que para produzir a equação do plano é preciso de um ponto $\in \pi_{f} \mathrm{e}$ de seu vetor normal $\left(\vec{n}_{f}\right)$, definiu-se o ponto como sendo a posição $p_{\text {hip_center }}$ e $\vec{n}=$ $\left(p_{\text {hip_right }}-p_{\text {hip_center }}\right) \times\left(p_{\text {hip_left }}-p_{\text {hip_center }}\right)$, onde $\times$ é a operação de produto vetorial.

No entanto, esse plano não pertence ao plano de visualização $2 \mathrm{D}\left(\pi_{v}\right)$ do cenário, que considera como normal o vetor $\overrightarrow{n_{v}}=(0,0,-1)$. Para projetar cada ponto de interesse $\left(p^{i}\right)$ de forma coerente com o plano de visualização, deverão ser realizadas outras operações, definidas na seguinte estratégia: 1) definir o eixo formado entre o $\pi_{f}$ e o $\pi_{v} ; 2$ ) encontrar o ângulo formado entre esses dois planos; 3) projetar $p^{i}$ no plano de projeção frontal $\left(\pi_{f}\right)$, adquirindo $p_{\text {proj }}^{i}$; e 4) tratar de rotacionar $p_{\text {proj }}^{i}$ de acordo com o eixo e ângulo encontrados, obtendo assim o ponto em $\pi_{v}$. Logo, foram duas operações de projeção em $p^{i}$, uma para projetar no plano frontal e outra para projetar no plano de visualização 2D.

Em 1) utiliza-se o cálculo de interseção entre dois planos, desde que $\overrightarrow{n_{f}}$ e $\overrightarrow{n_{v}}$ não sejam paralelos, existe uma reta de interseção. Que pode ser calculada por meio de um sistema com as equações dos dois planos.

O 2) é resolvido utilizando o cálculo do ângulo entre as normais, ou seja, utilizando o produto escalar entre os vetores normais unitários dos planos: $\arccos \left(\overrightarrow{n_{f}} \cdot \overrightarrow{n_{v}}\right)$.

Visto que cada $p^{i}$ deve ser projetado ortogonalmente ao plano $\pi_{f}$, calcula-se uma reta que seja perpendicular ao plano $\pi_{f}$ que passe pelo ponto $p^{i}$. Encontrar essa reta é simples, pois já se sabe de um ponto pertencente a reta $\left(p^{i}\right)$ e sua direção $\left(\overrightarrow{n_{f}}\right)$. Sendo assim, para resolver 3) utiliza-se resultado do cálculo da interseção entre essa reta e o plano $\pi_{f}$ cujo resultado é ponto $p_{\text {proj }}^{i}$.

Por fim, o 4) é resolvido utilizando o eixo encontrado em 1), como eixo arbitrário, e a posição que se deseja rotacionar, $p_{\text {proj }}^{i}$, que será rotacionado no ângulo definido em 2). Com isso o ponto projetado, $p_{\text {proj }}^{i}$, será inserido no plano de visualização seguindo um arco descrito pelo ângulo entre $\overrightarrow{n_{f}}$ e $\overrightarrow{n_{v}}$ obtendo-se o $p_{\text {view }}^{i}$ correspondente.

De forma análoga as operações são feitas para a projeção sagital. Sem esse tratamento dos dados a visualização fica comprometida aparentando desalinhamento entre alguns pontos de interesse.

Nesta visualização, também realizou-se a translação das posições das juntas projetadas no plano $\pi_{v}$ para uma posição desejada, fazendo com que a postura do usuário permaneça no "mesmo lugar". Para isso calculou-se o centro de massa das juntas de 
interesse $\left(p_{\text {com }}\right)$ diminuímos pela posição de âncora desejada $\left(p_{\text {anchor }}\right)$ e aplicou-se essa diferença em cada posição da junta ( $p_{\text {view }}^{i}$ ) de acordo com os cálculos:

$$
p_{\text {com }}=\frac{\sum_{i=0}^{8} p_{\text {view }}^{i}}{8}
$$

considerando oito juntas. Calculou-se então a nova posição para cada ponto projetado transladado:

$$
p_{\text {move }}^{i}=p_{\text {view }}^{i}-\left(p_{\text {com }}-p_{\text {anchor }}\right)
$$

A partir desta visão é possível aferir os ângulos entre o $p_{\text {move }}^{i}$ e eixos pré-definidos e assim observar algum possível problema de postura ou realizar o acompanhamento postural do usuário. Essas duas últimas ações não fazem parte do escopo deste trabalho.

\subsection{Cálculo dos Ângulos de Interesse}

Realizou-se o cálculo dos ângulos de interesse, com geometria analítica, por meio do produto escalar entre dois vetores definidos a partir da posição das juntas, $p_{\text {move }}^{i}$.

Utilizando-se deste cálculo aferiu-se quatro ângulos a partir da visualização frontal e um ângulo a partir da visão sagital. Na frontal, foi calculado o menor ângulo formado entre o vetor que forma uma linha horizontal do pescoço e o vetor formado da posição do pescoço ao ombro direito, da mesma forma em relação ao ombro esquerdo. Ainda no mesmo plano frontal, calculou-se o menor ângulo formado entre o vetor que forma

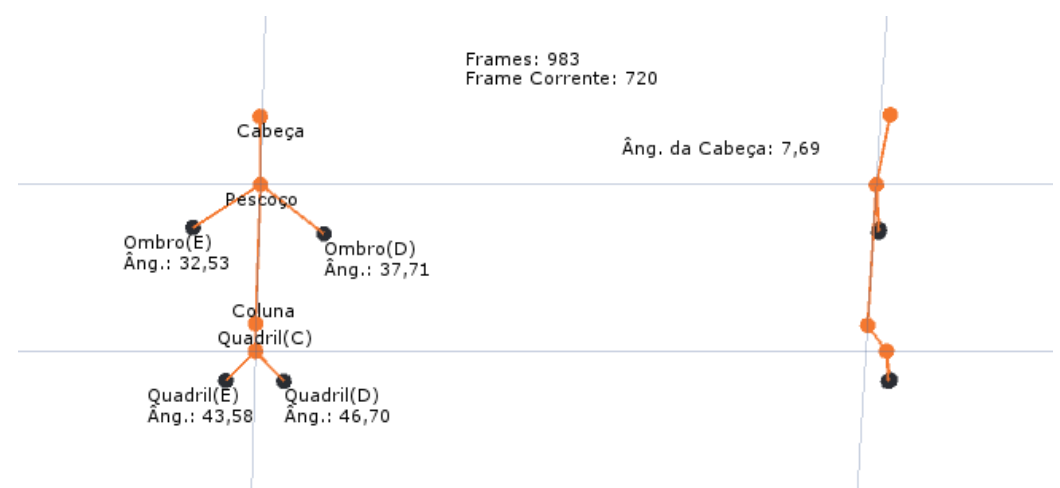

(a) Visualização 2D da projeção com tratamento seguindo eixo arbitrário e ângulo entre os planos de projeção.

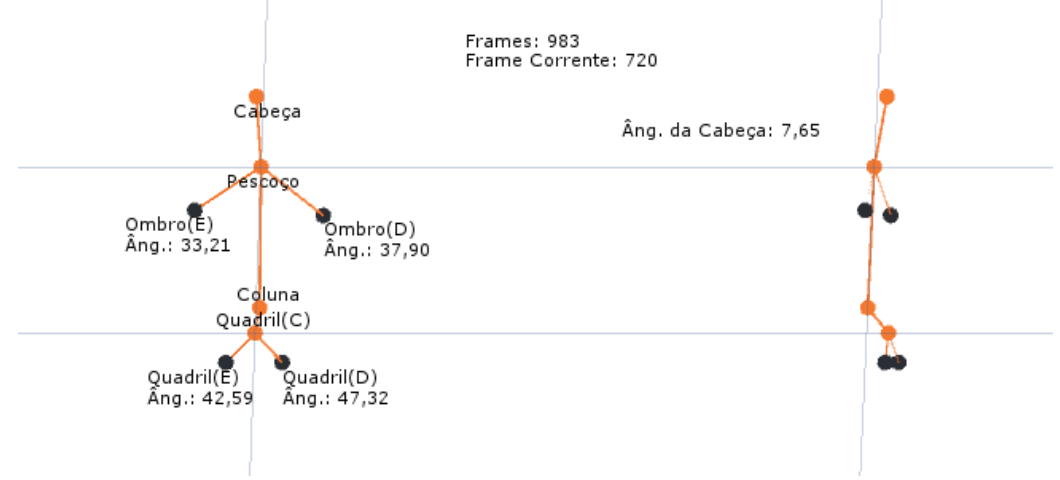

(b) Visualização 2D da projeção direto.

Figura 3. Comparação entre as duas projeções 2D. Utilizando tratamento da projeção que utiliza o ângulo formado entre os planos de projeção. 
uma linha horizontal com o centro do quadril com o quadril direito, da mesma forma em relação ao quadril esquerdo. E por fim, na visão sagital, calculou-se o ângulo formado entre a linha da coluna com a posição da cabeça. Os resultados dos cálculos podem ser observados na Figura 3a e na Figura 3b.

\section{Resultados e Discussão}

Este trabalho limitou-se a apresentar um método de visualização da postura, como realizado em um exame de postura tradicional, porém o usuário sendo livre de contexto, ou seja, não é necessária a definição de uma determinada postura como padrão, e também não é preciso realizar o toque para identificar as principais juntas, tornado-se então uma possível forma prática de avaliação postural. Os resultados da visualização dos cenários está presente no vídeo de referência (https : / youtu . be / j7bIuHXaBQY).

A contribuição principal do método está em planificar a postura capturada por um sensor de profundidade comparando com a avaliação postural realizada por métodos tradicionais. Nos modelos tradicionais além do paciente ter que utilizar roupas mais curtas e coladas o mesmo precisa ficar em uma posição padronizada por um determinado tempo, o que pode acarretar em desvios na sua postura de costume. Com este método é possível capturar a posição de uma pessoa em poses, além das tradicionais, para aferir a postura em comportamentos diários e não tratados por meio do simetrógrafo, como ao caminhar, usar o computador ou o smarthphone. Outra comparação pode ser feita por métodos que utilizam como base a fotogrametria computadorizada que utiliza imagens processadas para calculo da postura [Iunes et al. 2009]. Como o método apresentado já extrai posições de interesse diretamente e em tempo real por captura não é necessário um tratamento por imagem.

Na Figura 3, pode-se observar a diferença entre a projeção adquirida via o método proposto e utilizando a projeção direta sem o tratamento apresentado que projeta de acordo com o ângulo entre os planos. Observe que na Figura $3 b$ a posição dos ombros parece estar desalinhada, mas na realidade o usuário não tem problemas no alinhamento dos ombros, sendo assim o resultado mais realístico com o movimento capturado pode ser observado na Figura 3a.

\section{Conclusão e Trabalhos Futuros}

O presente trabalho preocupou-se em salientar o quanto são sensíveis os problemas relacionados a uma má postura, podendo inclusive comprometer todo modo de vida de uma pessoa. Neste sentido, realizou-se uma investigação sobre os métodos de verificar a postura e como utilizar recursos computacionais nesta tarefa. Foi identificado como recurso o sensor Kinect que, apesar de ser um equipamento barato, é capaz de auxiliar muitas aplicações, principalmente relacionadas à captura de movimentos.

Em conjunto com o ambiente de programação Unity e utilizando o sensor Kinect, projetou-se uma aplicação capaz de armazenar dados de captura, visualizar os mesmos de forma tridimensional e visualizar em duas dimensões objetivando aferir de alguma forma a postura e assim acompanhar a evolução do quadro clínico do paciente (não demonstrado neste trabalho). O método proposto independe do sensor utilizado, visto que se recorreu ao uso de geometria analítica para projetar as posições tridimensionais em um plano 2D independentemente da posição original do usuário. 
Pretende-se a partir desses dados e visualização dos ângulos, realizar testes para analisar a acurácia e aplicabilidade do método, bem como o procedimento de instalação do ambiente e assim criar um produto que possa ser utilizado por profissionais da área de fisioterapia e afins como uma alternativa de baixo custo e útil para sociedade.

Embora o termo baixo custo possa ser visto como algo bastante genérico, observase que neste trabalho procurou-se utilizar um equipamento que estava disponível para os testes do método e que não teve como foco realizar uma análise a cerca de qualidades de sensores Kinect, e que os trabalhos escolhidos estão inteiramente associados com essa temática. Ou seja, o método funciona com qualquer tipo de sensor que possa capturar posições de juntas de interesse para então projetar em um dispositivo gráfico usando duas visões, não sendo necessário recorrer a equipamentos de alto custo para isso.

\section{Referências}

Andersen, M. R., Jensen, T., Lisouski, P., Mortensen, A. K., Hansen, M. K., Gregersen, T., and Ahrendt, P. (2012). Kinect depth sensor evaluation for computer vision applications. Aarhus University, pages 1-37.

de Gusmão Lafayette, T. B., Teixeira, J. M. X. N., and Da Gama, A. E. F. (2019). Hybrid solution for motion capture with kinect v2 to different biotypes recognition. In XXVI Brazilian Congress on Biomedical Engineering, pages 249-259. Springer.

de Matos, O. (2011). Avaliação postural e prescrição de exercícios corretivos. Phorte Editora LTDA.

dos Santos, J. B. (2018). Descrição do método de avaliação postural de portland state university. Fisioterapia Brasil, 6(5):392-395.

Hoy, D. (2014). The global burden of low back pain: estimates from the global burden of disease 2010 study. Ann Rheum Dis. doi, 10.

Iunes, D., Bevilaqua-Grossi, D., Oliveira, A., Castro, F., and Salgado, H. (2009). Análise comparativa entre avaliação postural visual e por fotogrametria computadorizada. $R e$ vista Brasileira de Fisioterapia, 13(4):308-315.

Rumen, F. (2013). Kinect with ms-sdk. https://rfilkov.com/2013/12/16/ kinect-with-ms-sdk/.

Shum, H. P. H., Ho, E. S. L., Jiang, Y., and Takagi, S. (2013). Real-time posture reconstruction for microsoft kinect. IEEE Transactions on Cybernetics, 43(5):1357-1369.

Xu, Z., Zhang, Y., Fu, C., Liu, L., Chen, C., Xu, W., and Guo, S. (2020). Back shape measurement and three-dimensional reconstruction of spinal shape using one kinect sensor. In 2020 IEEE 17th International Symposium on Biomedical Imaging (ISBI), pages $1-5$. IEEE. 